\title{
REVIEW PAPER ON ANALYSIS OF VAPOUR ABSORPTION REFRIGERATION SYSTEM
}

\author{
Shubham Srivastava ${ }^{1}$, Ravi Kumar Sen $^{2}$, Arpit Thakur ${ }^{3}$, Manish Kumar Tated ${ }^{4}$ \\ ${ }^{1}$ M.Tech, Mechanical Engineering, Lovely Professional University, Punjab, India \\ ${ }^{2}$ M.Tech, Mechanical Engineering, Lovely Professional University, Punjab, India \\ ${ }^{3}$ M.Tech, Mechanical Engineering, Lovely Professional University, Punjab, India \\ ${ }^{4}$ M.Tech, Mechanical Engineering, Lovely Professional University, Punjab, India
}

\begin{abstract}
At the present time refrigeration plays a very important role in our daily as well as industrial life. But due to some problems for example, refrigerant the CFC is also affecting our ecosystem very rapidly. So there is need of working on these systems in order to avoid such harms. Vapour Absorption Refrigeration System acts as an alternative to existing systems as it requires low grade of energy for example; solar energy as it's renewable and present in enormous amount. So, in recent times a lot of research is going on to improve such systems that affects least to the environment and humanity but acts for same purpose in very efficient manner.
\end{abstract}

Keywords: VARS, Refrigeration Cycle, $\mathrm{NH}_{3}-\mathrm{H}_{2} \mathrm{O}, \mathrm{COP}, \mathrm{CFC}$

\section{INTRODUCTION}

In our daily life whenever the term "refrigeration" comes in our mind; we simply think of refrigerator that works on the electrical supply and thus provides us chilled water, ice and preserves our beverages and eatables from getting exhausted. In these types of refrigerators the components that are used are "compressor, condenser, and evaporator and expansion valves". These different components plays different role but all of them are so very much needed in order to complete the refrigeration cycle. Fig-1describes schematic cycle description of vapour compression system. The compressor is utilized for the purpose of compressing low pressure and temperature vapour refrigerant to high pressure and temperature. In condenser the high pressure and temperature vapour refrigerant is cooled and condensed. In during this process, the refrigerant gives up its latent heat to the surrounding medium. Now in evaporator, liquidvapour refrigerant is transformed into low pressure and temperature refrigerant at low pressure and temperature by absorbing the latent heat from the medium. At the end, comes role of expansion valves whose function is to allow to liquid refrigerant under high pressure and temperature to lower down its temperature and pressure so as to feed into the evaporator. Thus; in these steps the complete refrigeration cycle is completed and hence the net refrigeration effect is achieved [1]. There is also a term used "refrigerant" used which is circulated among these four components and which is the most essential need of the complete system. In common usage CFC is used as a refrigerant in the above described refrigeration system. The limitation related to it is that when it leaks into the atmosphere it depletes our ozone layer, which is keeping us safe and secured from the ultraviolet rays of the sun.
Here; COP is given by the relation;

$$
\text { COP }(V C R S)=\frac{\text { Refrigeration Effect }}{\text { Work Done }}
$$

So now, in order to avoid this harm which is happening in our environment researchers are working on another type of refrigerating system known as "VARS i.e. VAPOUR ABSORPTION REFRIGERATION SYSTEM" and is system is somewhat different from the used one's. The difference among the two systems are:- Firstly; in first system there isa compressor where as in VARS systemthere is no compressor and it's role is played bycombination of "absorber, generator and expansion valve". Secondly; in VCRS system there is high grade of energy" is needed for performance of the system whereas in VARS system "low grade of energy" is utilized for example: solar energy, geothermal energy, wind energy and etc. Now in this paper we are going to take into account "solar energy" as it is renewable and available in large amount.

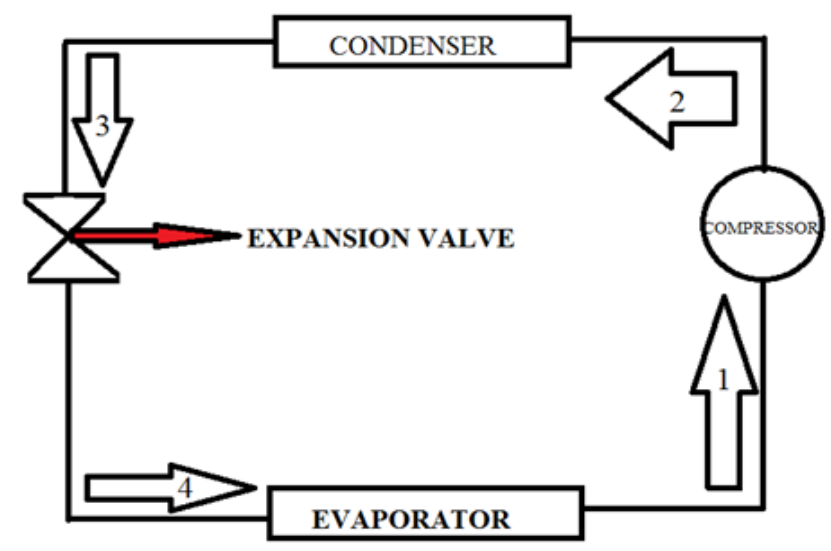

Fig-1: Simple Vapour Compression System 


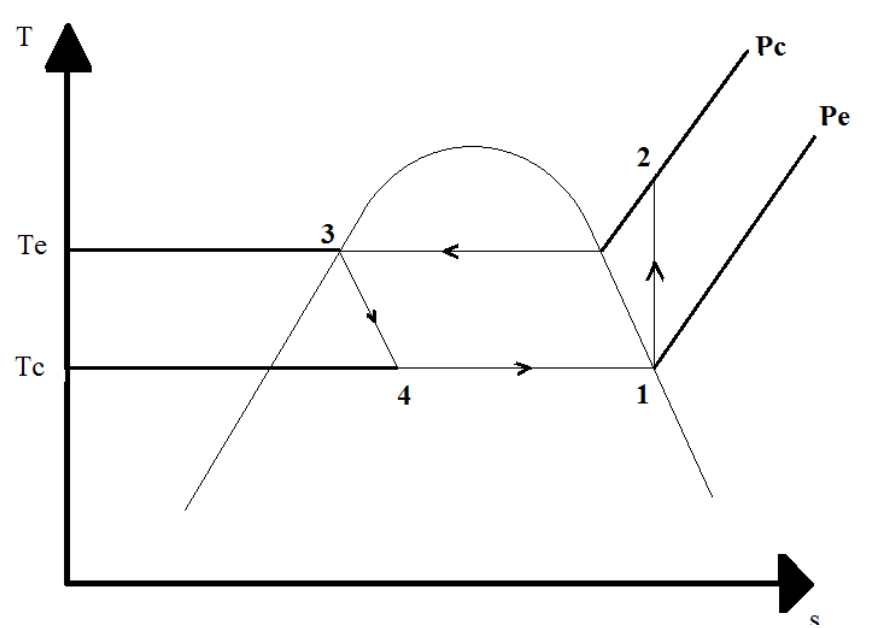

Fig-2: T-s plot for Vapour Compression System

\section{CYCLE DESCRIPTION}

Fig-3 depicts the schematic diagram of vapour absorption system. In such systems, low pressure refrigerant vapour leaves the evaporator and enters the absorber. Here the formation of "solution pair" takes place i.e. combination of refrigerant and absorbent and the formed solution is strong in nature. Now this strong solution is then pumped to the generator and here the pressure increases. In generator this strong solution is heated by someexternal source whichinthis case study is "solar energy". After the heating process is accomplished strong solution at high pressure moves to the condenser leaving back the weak solution which is send back to the absorber using an expansion valve. Now the high pressure refrigerant moves from generator to the condenser where the refrigerant vapour is condensed to high pressure liquid refrigerant. This liquid refrigerant is passed to the expansion valve and where it is forwarded to the evaporator, where the refrigeration effect is achieved and thus completes the complete vapour absorption cycle [2].

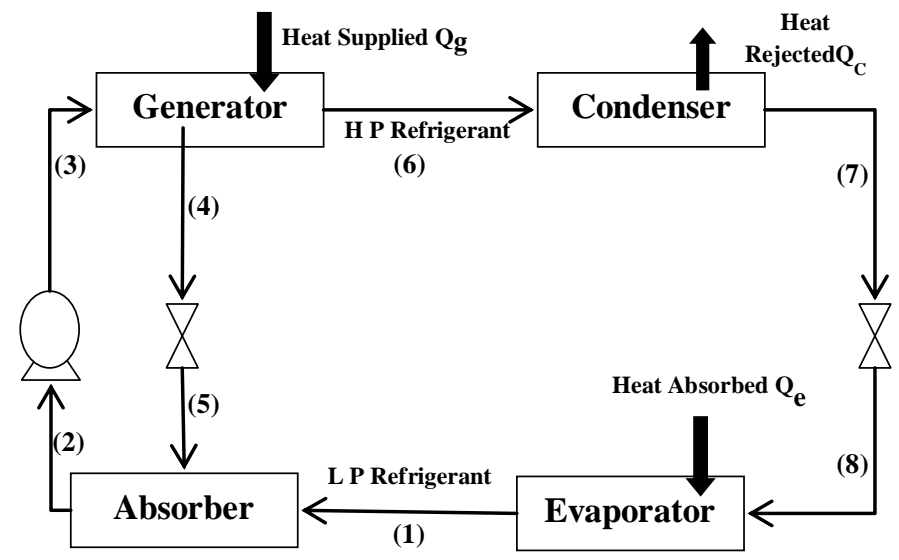

Fig-3: Simple Vapour Absorption System

Here;

'H P' stands for "High Pressure", 'L P' stands for "Low Pressure".
In this type of system the refrigerant is not single handedly used despite there is another substance used in combination with refrigerant and that is known as "absorbent" and they both when are combined is called a "solution pair". For example: $\mathrm{NH}_{3}-\mathrm{H}_{2} \mathrm{O} \& \mathrm{LiBr}-\mathrm{H}_{2} \mathrm{O}$. In the first one ammonia $\left(\mathrm{NH}_{3}\right)$ act as "refrigerant" and water $\left(\mathrm{H}_{2} \mathrm{O}\right)$ acts as "absorbent" whereas in second case lithium bromide $(\mathrm{LiBr})$ acts as "absorbent" and $\left(\mathrm{H}_{2} \mathrm{O}\right)$ acts as "refrigerant".

In during the selection of solution pairs several thermodynamic requirements [1] are need to be fulfilled and that are:

a) Solubility Requirement: The refrigerant should have more solubility in the absorbent such that a strong solution must be highly rich in refrigerant.

b) Boiling Point Requirement: In among the components of solution pair i.e. refrigerant and absorber, the difference in boiling point must be atleast of approximately $200^{\circ} \mathrm{C}$ thus only absorbent free refrigerant is boiled off from the generator.

c) The viscosity factor should be least in order to minimize pump work.

They should have excellent chemical and thermal stability and also low specific heat etc.Now the running cost of such systems are given by the term "COP i.e. Coefficient of Performance"

$$
C O P=\frac{Q e}{Q g}
$$

Here; $\mathrm{Q}_{\mathrm{e}}$ is the heat absorbed by the absorber.

$A n d Q_{g}$ is the amount of heat supplied to the generator. 2.1 Derivation of Maximum COP in VARS

In Fig-4 we can easily observe the transfer of energy taking in the entire system among the components. Now, let's start with understanding the working of the system;

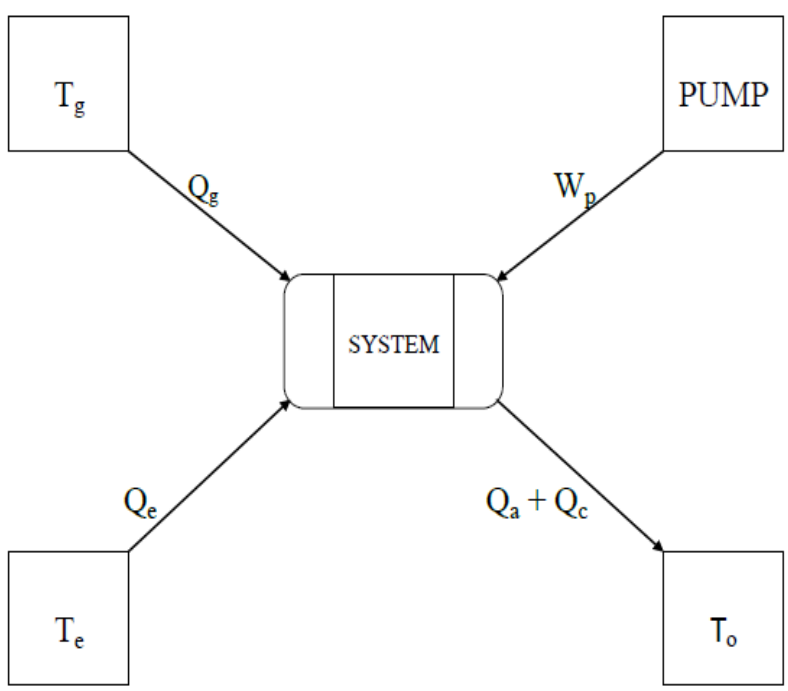

Fig-4: Various Energy Transfers in a Vapour Absorption Refrigeration System 
From the first law of thermodynamics;

$$
\mathrm{Qg}+\mathrm{Qe}-\mathrm{Q}(\mathrm{c}+\mathrm{a})+\mathrm{Wp}=0
$$

Where; $\mathrm{Q}_{\mathrm{e}}$ is the heat transferred to the absorption system at evaporator temperature $T_{e}, Q_{g}$ is the heat transferred to the generator of the absorption system at temperature $T_{g}, Q_{a+c}$ is the heat transferred from the absorber and condenser of the absorption system at temperature $\mathrm{T}_{\mathrm{o}}$ and $\mathrm{W}_{\mathrm{p}}$ is the work input to the solution pump. From the second law of thermodynamics;

$$
\Delta s(\text { total })=\Delta s(\text { sys })+\Delta s(\text { surr. }) \geq 0
$$

where $\Delta s$ (total) is the total entropy change which is equal to the sum of entropy change of the system $\Delta s$ (sys) and entropy change of the surroundings $\Delta s$ (surr.) Since the refrigeration system operates in a closed cycle, the entropy change of the working fluid of the system undergoing the cycle is zero, i.e., $\Delta s$ (sys) $=0$.

The entropy change occurring in the surrounding is given by:

$$
\Delta s(\text { surr. })=\frac{-\mathrm{Qe}}{T e}-\frac{\mathrm{Qg}}{T g}+\frac{\mathrm{Q}(\mathrm{a}+\mathrm{c})}{T o}
$$

Substituting the expression for first law of thermodynamics i.e. eqn. (3) in the above eqn. (5);

$$
\mathrm{Q} g\left(\frac{T g-T o}{T g}\right) \geq \mathrm{Qe}\left(\frac{\mathrm{To}-\mathrm{Te}}{\mathrm{Te}}\right)-W p
$$

Neglecting pump work $\left(\mathrm{W}_{\mathrm{p}}\right)$ the COP is given by:

$$
C O P(V A R S)=\frac{\mathrm{Qe}}{\mathrm{Qg}} \leq\left(\frac{T e}{T o-T e}\right)\left(\frac{T g-T o}{T g}\right)
$$

From the above equation it can be observed that COP of ideal VARS system is equal to the product of:

$>$ Carnot heat engine efficiency working between temperature $\mathrm{T}_{\mathrm{g}}$ and $\mathrm{T}_{\mathrm{o}}$

$>$ Carnot refrigeration system between $\mathrm{T}_{\mathrm{o}}$ and $\mathrm{T}_{\mathrm{e}}$

And so has been represented in the Fig. 5

$$
\begin{aligned}
& \operatorname{COP} \text { ideal }(\text { VARS })=\frac{\mathrm{Qe}}{\mathrm{Qg}} \leq\left(\frac{T e}{T o-T e}\right)\left(\frac{T g-T o}{T g}\right) \\
= & \operatorname{COP}(\text { carnot }) * \eta(\text { carnot })
\end{aligned}
$$

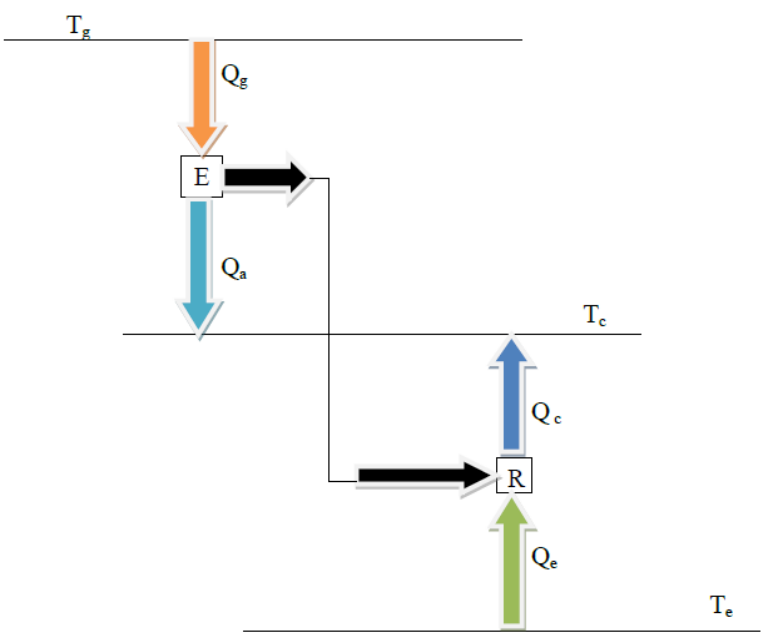

Fig-5: VARS as combination of engine and refrigerator

\section{APPLICATIONS}

This system has wide range of application like food storage and also for vaccine storage. In real, this technology can be used in rural areas where the accommodation of electricity is impossible. Uppal et al. [3] in 1986 constructed a small capacity solar-powered $\mathrm{NH}_{3}-\mathrm{H}_{2} \mathrm{O}$ absorption refrigerator to store vaccines in remote locations. In 1993, Sierra et al. [4] used a solar pond to power an intermittent absorption refrigerator with $\mathrm{NH}_{3}-\mathrm{H}_{2} \mathrm{O}$ solution. It is reported that generation temperatures as high as $73^{\circ} \mathrm{C}$ and evaporation temperatures as low as $-2^{\circ} \mathrm{C}$ could be obtained. The COP under such working conditions was in between 0.24-0.28. Anyanwu and Ezekwe [5] also tested a solid absorption solar refrigerator using activated carbon-methanol as the working solution pair. Critoph [6] studied a rapid cycling solar/biomass-powered adsorption refrigeration system with activated carbon-ammonia as working pair. The thermal COP was about 0.3 when the initial generator temperature was about $50^{\circ} \mathrm{C}$ and evaporating temperature was about $0^{\circ} \mathrm{C}$.

\section{RESULTS AND CONCLUSION}

From the previous study it's now quite clear that Vapour Absorption System has an upper edge over the VapourCompression System taking in regard of the environment. In absorption systems there is no danger of depletion of ozone layer, all renewable sources of energy could be used such as, solar energy, wind energy, bio-gas etc. The system is also free compressor less, as a result of which the life span of system is longer than vapour compression one's. The only disadvantage with such system is low $\mathrm{COP}$ and requires longer time duration to perform the complete operation. Till date two "solution pairs" have been utilised in such systems and that are " $\mathrm{NH}_{3}-\mathrm{H}_{2} \mathrm{O} \& \mathrm{LiBr}$ $\mathrm{H}_{2} \mathrm{O}$ " and further research is going to in order to find some more alternate solution pairs that would $b$ effective in during its operation and produces same amount of refrigeration effect. Some of them are "Ammonia Sodium Thiocyanate $\left[\mathrm{NH}_{3}-\mathrm{NaSCN}\right]$, Ammonia Lithium Nitrate $\left[\mathrm{NH}_{3}-\mathrm{LiNO}_{3}\right]$, carbon methanol and many more. 


\section{ACKNOWLEDGEMENTS}

In this section, I would acknowledge the efforts of mine coauthors i.e. "RAVI KUMAR SEN, ARPIT THAKUR, MANISH KUMAR TATED" as because along with mine their hard work and dedication the paper is finally ready. I would also feel proud to acknowledge the efforts of mine worthy mentor "MR. SUDHANSHU DOGRA" as with his guidance it was only possible for us to complete the paper within time limit and also with proper validation.

\section{REFERENCES}

[1]. Khurmi, R.S. (2012). Refrigeration \& Air-Conditioning 6th ed. New Delhi: S. Chand.

[2]. Y. Fan, L. Luo, B. Souyri. (2007). Review of solar sorption refrigeration technologies: Development and applications. ELSEVIER, 1758-1775.

[3]. Uppal AH, Norton B, Probert SD. A low-cost solarenergy stimulated absorption refrigerator for vaccine storage. Applied Energy 1986;25:167-74.

[4]. Sierra FZ, Best R, Holland FA. Experiments on an absorption refrigeration system powered by a solar pond Heat Recovery System CHP 1993;13:401-8.

[5]. Anyanwu EE, Ezekwe CI. Design, construction and test run of a solid absorption solar refrigerator using activated carbon/methanol, as working fluid pair. Energy Conversion Manage 2003;44:2879-92.

[6]. Critoph RE. Rapid cycling solar/biomass powered adsorption refrigeration system. Renewable Energy 1999;16:673-8.

\section{BIOGRAPHIES}

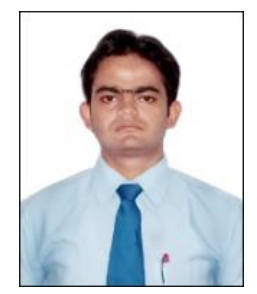

I am ShubhamSrivastava, born in Varanasi, Uttar Pradesh, pursuing M.Tech in Mechanical Engineering with specialization in [Thermal Department]. My area of intrest is in "Energy Resources \& Refrigeration and have also submitted papers in conferences.

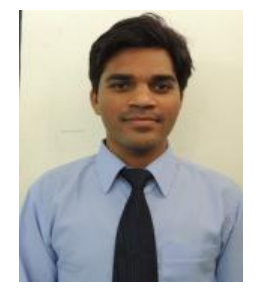

I am Ravi Kumar Sen, born in Chhattisgarh, pursuing M.Tech in Mechanical Engineering with specialization in [Thermal Department.]. I have a publication on " $3 \mathrm{D}$ CFD analysis of passive techniques in shell and tube heat exchanger".

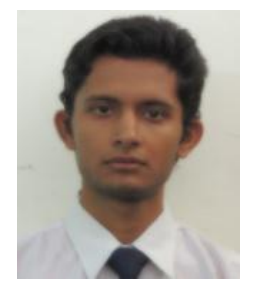

I am Arpit Thakur, born in Ajmer, Rajasthan, pursuing M.Tech in Mechanical Engineering with specialization in [Thermal Department.]. I have a publication on the "ground coupled heat exchanger using CFD approach".

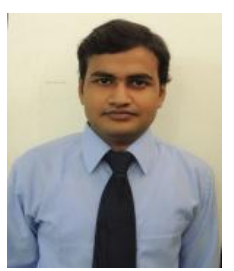

I am Manish Kumar Tated, born in Chhattisgarh, pursuing M.Tech in Mechanical Engineering with specialization in [Thermal Department.]. I have a publication on the "study of double pass solar air heater". 